\title{
Demoralization in Opioid Dependent Patients: A Comparative Study with Cancer Patients and Community Subjects
}

\author{
Cor A.J. De Jong ${ }^{*}, 1,2$, David W. Kissane ${ }^{3}$, Ronit J. Geessink ${ }^{2}$ and Dieke van der Velden² \\ ${ }^{I}$ Novadic-Kentron, Network for Addiction Treatment Services \\ ${ }^{2}$ Nijmegen Institute for Scientist-Practitioners in Addiction, Radboud University Nijmegen, P.O. Box 9104, $6500 \mathrm{HE}$ Ni- \\ jmegen, The Netherlands \\ ${ }^{3}$ Department of Psychiatry and Behavioral Sciences, Memorial Sloan-Kettering Cancer Center, New York, NY 10021, USA
}

\begin{abstract}
Aim: To study existential distress or demoralization expressed as meaninglessness and helplessness in opioid dependent patients.

Method: Comparison of existential distress between opioid dependent patients $(n=131)$, patients with advanced cancer $(\mathrm{n}=100)$ and a community based sample without severe psychiatric or somatic disorders $(\mathrm{n}=190)$ as measured with the Demoralization Scale.

Results: Community controls without somatic or psychiatric disorders have significantly lower scores on all Demoralization Subscales. Opioid dependent patients are strikingly more demoralized than patients with cancer.

Conclusion: Opioid dependent patients suffer from severe existential distress (meaninglessness, helplessness) which can explain the high prevalence of suicide in this group.
\end{abstract}

Keywords: Opioid dependence, addiction, advanced cancer, existential distress, demoralization.

\section{INTRODUCTION}

Addiction is more and more considered to be a chronic brain disease with a destructive lifestyle in which there is often loss of the meaning and purpose of life [1]. These two negative psychological aspects of addiction fit well in the concept of demoralization. As stated by Jerome Frank [2] demoralization can result from persistent failure to cope with internally or externally induced stresses that the person and those close to him expect to handle. It is characterized by feelings of impotence, isolation and despair. The person's self-esteem is damaged and he feels rejected by others. Alienation may contribute to a sense of meaninglessness of life. Patients seldom ask for help because of an existing disease only. They seek help because of the accompanying demoralization caused by their inability to solve problems [3]. This makes the construct of demoralization important in modern medicine, including addiction medicine.

Demoralization comes up if persons are confronted with their inability to handle problems and covers a broad spectrum with disheartenment on the one and despair on the other end. Disheartenment is most characteristic of this mental state, but next to it are feelings of shame, anger, anxiety and alienation [4]. The phenomenology of demoralization in the medically ill is characterised by non-specific dysphoria, disheartenment, loss of confidence and development of subjective incompetence, loss of meaning, hopelessness and helplessness, social disconnectedness and the desire to die.

*Address correspondence to this author at the Nijmegen Institute for ScientistPractitioners in Addiction, Radboud University Nijmegen, P.O. Box 9104, 6500 HE Nijmegen, The Netherlands; E-mail: c.dejong@acsw.ru.nl
Demoralization is a chronic state of mind distinct from acute distress, failure to cope, depression and general uncertainty about the future and can be regarded best as a general descriptor of chronic psychological distress. In describing demoralization this way it is synonymous with suffering or existential distress. This is in line with the original description of the concept by Jerome Frank.

Existential distress or demoralization is under investigated in opioid-dependent patients in comparison with other patient groups. In the care of patients with cancer and other advanced diseases, insight into existential distress has proved crucial to optimizing clinical response [5]. Helplessness and meaninglessness - expressions of existential distress - are important predictors for suicide [6] as are substance related disorders [7].

Existential distress can be measured by a self report questionnaire that comprises loss of meaning and purpose, dysphoria, disheartenment, helplessness and sense of failure $r$ and captures demoralization reliably and validly [4].

Addicted patients frequently provoke a multiplicity of feelings, thoughts and reactions in health care providers because of their complex interactions during treatment or disease management $[8,9]$. The countertransference reactions on the complex behavior of addicted patients including their expression of existential distress can distance the clinician from the patient, with potential abandonment, which can augment the risk of suicide. Taking addiction as a disease, people afflicted by it are ill and should be treated as patients. In this treatment, more attention should be given to chronic existential distress as one of the potential sources of negative countertransference reactions. 
The aim of this study is to explore chronic existential stress or demoralization in opioid dependent patients in long term methadone maintenance treatment. As far as we know, there are no comparative studies examining demoralization in substance dependent patients. We sought to examine this in opioid dependent patients, and to compare their demoralization with patients with advanced cancer and a group of community-based subjects without serious somatic or psychiatric disorders.

\section{METHOD}

\section{Participants}

The opioid-dependent subjects were from a Methadone Maintenance Treatment (MMT) unit in the Netherlands taking part in a comprehensive study of psychiatric co-morbidity. Criteria for inclusion were MMT and willingness to provide informed consent. Patients unable to speech Dutch were excluded. Recruitment was from March to June 2006.

The study was approved by the Dutch 'Ethical Assessment Committee for Experimental Investigations on People'.

The consecutive series of patients with advanced cancer attended either the Pain and Palliative Care or PsychoOncology Clinics at the Peter MacCallum Cancer Institute $(n=63)$ and the Psycho-oncology clinic or Caritas Christi Hospice ward at St. Vincent's Hospital $(n=37)$ in Melbourne, Australia. Exclusion criteria were: confusion, as defined by a Mini Mental Sate Examination of $<24$; too unwell or frail; inability to read English; and psychosis or intellectual handicap. The institutional ethics committee gave approval for the study.

The community-based subjects were recruited by means of 'Snowball Sampling' [3] in the social network of collaborators of the research institute NISPA. The potential participants received a 'participant's letter' describing the purpose and significance of the project. Exclusion criteria were current treatment for somatic or psychiatric disorders. Two hundred and five questionnaires were sent, with a response rate of $92.7 \%$.

\section{Instrument}

The Demoralization Scale [4] (DS) is a self report questionnaire consisting of 24 statements (for instance 'I no longer feel emotionally in control') that should be answered on a five point Likert scale ranging from 'never' to 'all the time'. The DS has five subscales describing (1) loss of meaning and purpose, (2) dysphoria, (3) disheartenment, (4) helplessness and (5) sense of failure. The English version of the DS showed good reliability and validity. The subscales demonstrate satisfactory internal coherence, yet sufficient differentiation from one other. Convergent validity was shown between the DS and other measures for existential distress, hopelessness, depression and a desire to die. The DS was able to differentiate a subset of patients who are demoralized and yet not clinically depressed.

Three translators independently translated the DS into Dutch. These were compared with each other to reach consensus about item translation. The resultant consensus version was back-translated into English by a fourth, bilingual psychologist. Differences between original and back-translated items were reviewed by the scale's originator (DWK) and adjusted until the original meaning had been preserved.
The psychometric properties of the Dutch version were comparable with the English version [10] in the community based sample as well as in the opioid dependent group of patients. The Dutch version also pointed at an overlap between demoralization and acute distress, failure to cope, depression and general uncertainty about the future, but the extent of the overlap was such that demoralization should be regarded as a construct distinct from these other psychological constructs.

\section{Statistical Analysis}

All quantitative data were entered and analyzed using SPSS (SPSS, Inc., Chicago, IL). To test for differences between the cohorts on the five subscales and the total score of the DS, ANOVAs with post hoc comparisons for observed means (Bonferroni) were performed.

\section{RESULTS}

Socio-demographic characteristics of the three cohorts and five dimensions of the Demoralization Scale are shown in Table 1. It is clear that the three populations differ with respect to age and gender. The community group without somatic or psychiatric disorders has significantly lower scores on all demoralization subscales, pointing at the level of existential distress in the two cohorts of patients. Opioid-dependent patients are noteworthy for higher scores on all subscales of the DS than cancer patients.

\section{DISCUSSION}

As far as we know, this is the first study that reports on the level of existential distress as measured with the Demoralization Scale in opioid dependent patients. As expected, the two clinical cohorts showed significantly higher scores on all subscales of the Demoralization Scale. Surprisingly, the opioid dependent patients proved to be significantly more demoralized than patients with advanced cancer.

One limitation of our study is that the design does not allow us to control for differences in the populations other than the aspects of demoralization. Any conclusions about these differences can only be tentative, but age, gender, the longer duration of illness and the frequently noticed lack of a social network in the opioid-dependent patients should be taken into account as confounders.

The differences in demoralization found in this study could be accounted for by cross-cultural differences in the populations studied. Although he psychometric evaluation of the Dutch version of the Demoralization Scale showed close resemblance with the original version cross-cultural differences could not be ruled out. Therefore further research is needed in homogenous populations such as opioid dependent patients in methadone maintenance treatment in different countries. Nevertheless, the size of differences is such that in our opinion conclusions should be drawn concerning the clinical importance of the degree of demoralization in the opioid dependent population in methadone maintenance treatment. Another limitation is that other psychological constructs or psychiatric disorders were not measured in the three groups in the same way, which made it difficult to explore the overlap and distinctions in detail. If demoralization is taken as a descriptor of general chronic distress the high level of distress found in opioid dependent patients is an important point of attention in the clinical encounter. 
Table 1. Means (SD) of Total Score and Subscales of the Demoralization Scale Compared Between a Community-Based Normative Sample and Cohorts of Opioid-Dependent and Cancer Patients Using Analysis of Variance

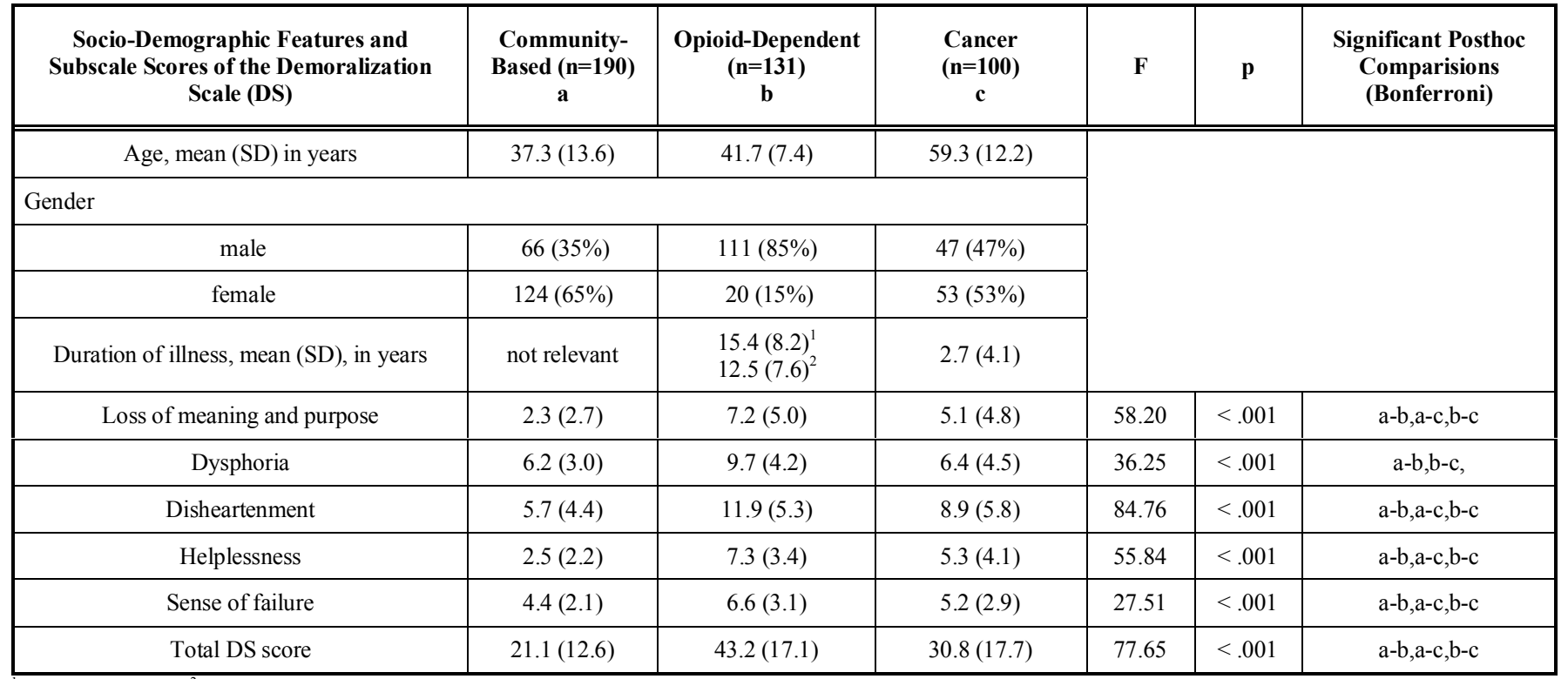

${ }^{1}$ Heroine use in years ${ }^{2}$ Methadone use in years.

Addiction is more and more regarded as a chronic brain disease with a complex biopsychosocial background including genetic vulnerability, early traumatisation, personality pathology and societal influences. In this complex interplay between biological, psychological and societal aspects of dependence and chronic states of mind the neurobiological aspects of a chronic demoralization and the association with the changes in the functions of the addicted brain are still to be elucidated.

In the consulting room therapists are confronted with demoralized addicted patients. The plight of opioid-dependent patients and their potential to become disheartened, develop meaninglessness, helplessness, dysphoria and a sense of failure is noteworthy - in short, they risk profound demoralization. Important consequences about the degree of demoralization found in this study include its possible negative impact on patients in their efforts to achieve a healthier lifestyle. But it also influences their relationships with professionals in addiction treatment programs, through transfer of their feelings of demoralization onto their therapists. Therapists dealing with severely demoralized opioid-dependent patients need to understand how their counter-transference impacts upon the working alliance and strive for the same sensitivity expected with other life threatening diseases. Addicted patients are not always easy to deal with because of their complex behavior in interpersonal encounters and they arouse different and often confusing feelings in therapists [8]. Demoralization adds to these complexity and can result in feelings of detachment or withdrawal in therapists. Suicide may be a tragic outcome if the existential distress or demoralization is not recognized and left untreated because of these kind of feelings in therapists.

\section{REFERENCES}

[1] Volkow ND, Li TK. Drugs and alcohol: treating and preventing abuse, addiction and their medical consequences. Pharmacol Ther 2005; 108(1): 3-17.

[2] Frank JD. Psychotherapy, the restoration of morale. Am J Psychiatry 1974; 131: 271-4.

[3] Stoffer R. Demoralization and the failed mission. In the footsteps of Jerome D Frank. J Psychother 2001; 27: 366-79.

[4] Kissane DW, Wein S, Love A, Lee XQ, Kee PL, Clarke DM. The Demoralization scale: a report of its development and preliminary validation. J Palliati Care 2004; 20(4): 269-76.

[5] Kissane DW, Clarke DM, Street AF. Demoralization syndrome - a relevant psychiatric diagnosis for palliative care. J Palliat Care 2001; 17: 12-21.

[6] Kuo WH, Gallo JJ, Eaton WW. Hopelessness, depression, substance disorder, and suicidality--a 13-year community-based study. Soc Psychiatry Psychiatr Epidemiol 2004; 39(6): 497-501.

[7] Tondo L, Baldessarini RJ, Hennen J, Minnai GP, Salis P, Scamonatti L. Suicide attempts in major affective disorder patients with comorbid substance use disorders. J Clin Psychiatry 1999; 60(Supp1 2) :639.

[8] Imhof JE. Overcoming countertransference and other attiduninal barriers in the treatment of substance abuse. In: Washton AM, Ed. Psychotherapy and substance abuse: A Practitioner's Handbook. The Guilford Press: New York 1995

[9] Forrest GG. Countertransference in chemical dependence counseling. The Haworth Press: New York 2002.

[10] Geesink RJ. Demoralization in a normal population: validation of the Demoralization scale. Master thesis, Radboud University Nijmegen, 2006.

This is an open access article licensed under the terms of the Creative Commons Attribution Non-Commercial License (http://creativecommons.org/licenses/by$\mathrm{nc} / 3.0 /<\mathrm{http}: / /$ creativecommons.org/licenses/by-nc/3.0/>) which permits unrestricted, non-commercial use, distribution and reproduction in any medium, provided the work is properly cited. 